\title{
Star Formation in Quasar Host Galaxies at Redshift 6: Millimeter Surveys and New Insights from ALMA
}

\author{
Ran Wang ${ }^{1,2,11}$, Jeff Wagg ${ }^{3}$, Chris L. Carilli ${ }^{1}$, Fabian Walter ${ }^{4}$, Xiaohui \\ Fan $^{2}$, Frank Bertoldi ${ }^{5}$ Dominik. A. Riechers ${ }^{6}$, Alain Omont ${ }^{7}$, Karl M. \\ Menten $^{8}$, Pierre Cox ${ }^{9}$, Michael A. Strauss ${ }^{10}$, and Desika Narayanan ${ }^{2}$ \\ ${ }^{1}$ National Radio Astronomy Observatory, PO Box 0, Socorro, NM, USA 87801, \\ email: rwang@nrao.edu \\ ${ }^{2}$ Steward Observatory, University of Arizona, 933 N Cherry Ave., Tucson, AZ, 85721, USA \\ ${ }^{3}$ European Southern Observatory, Alonso de Córdova 3107, Vitacura, Casilla 19001, Santiago \\ 19, Chile ${ }^{4}$ Max-Planck-Institute for Astronomy, Königsstuhl 17, 69117 Heidelberg, Germany \\ ${ }^{5}$ Argelander-Institut für Astronomie, University of Bonn, Auf dem Hügel 71, 53121 Bonn, \\ Germany ${ }^{6}$ Astronomy Department, Cornell University, 220 Space Sciences Building, Ithaca, \\ NY 14853, USA ${ }^{7}$ Institut d'Astrophysique de Paris, CNRS and Universite Pierre et Marie \\ Curie, Paris, France ${ }^{8}$ Max-Planck-Institut für Radioastronomie, Auf dem Hügel 71, 53121 \\ Bonn, Germany ${ }^{9}$ Institute de Radioastronomie Millimetrique, St. Martin d'Heres, F-38406, \\ France ${ }^{10}$ Department of Astrophysical Sciences, Princeton University, Princeton, NJ, USA, \\ 08544 \\ ${ }^{11}$ Jansky Fellow
}

\begin{abstract}
We have been carrying out a systematic survey of the star formation and ISM properties in the host galaxies of $\mathrm{z} \sim 6$ quasars. Our $250 \mathrm{GHz}$ observations, together with available data from the literature, yield a sample of $14 \mathrm{z} \sim 6$ quasars that are bright in millimeter dust continuum emission with estimated FIR luminosities of a few $10^{12}$ to $10^{13} \mathrm{~L} \odot$. Most of these millimeter-detected $\mathrm{z} \sim 6$ quasars have also been detected in molecular CO line emission, indicating molecular gas masses on order of $10^{10} \mathrm{M}_{\odot}$. We have searched for [C II] 158 micron fine structure line emission toward four of the millimeter bright $\mathrm{z} \sim 6$ quasars with ALMA and all of them have been detected. All these results suggest massive star formation at rates of about 600 to $2000 \mathrm{M}_{\odot} \mathrm{yr}^{-1}$ over the central few kpc region of these quasar host galaxies.
\end{abstract}

Keywords. quasars: general — galaxies: starburst — galaxies: evolution — submillimeter

\section{Introduction}

More than fifty quasars at $\sim 6$ have been detected from large optical and near-infrared surveys, such as the Sloan Digital Sky Survey (hereafter SDSS, e.g., Fan et al. 2006) and the Canada-France High redshift Quasar Survey (CFHQS, Willott et al. 2007). These objects represent the first generation of supermassive black holes (SMBHs) which formed within 1 Gyr of the Big Bang and are accreting at their Eddington limit (e.g. Kurk et al. 2007). Strong millimeter dust continuum, molecular CO, and [C II] $158 \mu \mathrm{m}$ fine structure line emission were first detected from the $\mathrm{z}=6.42$ quasar SDSS J114816.64+525150.3 (hereafter J1148+5251, Bertoldi et al. 2003a; Maiolino et al. 2005, 2012; Walter et al. 2003; Riechers et al. 2009), indicating intense star formation with a peak surface density of $\sim 1000 \mathrm{M}_{\odot} \mathrm{yr}^{-1} \mathrm{kpc}^{-2}$ over the central $1.5 \mathrm{kpc}$ region (Walter et al. 2009 ). The dynamical mass estimated with the resolved CO line emission indicates SMBH-bulge mass ratios more than one order of magnitude higher than the typical value found in the present universe (Walter et al. 2004). The millimeter observations of J1148+5251 suggest an 
early phase of SMBH-galaxy evolution and the dust continuum, molecular/atomic line emission have been searched in more quasars at the highest redshift (e.g. Carilli et al. 2007; Wang et al. 2008, 2011a, 2011b; Venemans et al. 2012). In this paper, we summarize recent millimeter observations of the sample of quasars known at $\mathrm{z} \sim 6$ and discuss the star forming activity in these earliest quasar host galaxies.

\section{Millimeter observations of the $\mathrm{z} \sim 6$ quasars}

A sample of 41 quasars at $\mathrm{z} \sim 6$ have been observed at $250 \mathrm{GHz}$ using the MAMBO bolometer array on the IRAM 30-m telescope (Bertoldi et al. 2003a; Petric et al. 2003; Willott et al. 2007; Wang et al. 2008, 2011a). This $250 \mathrm{GHz}$ observed sample includes all the most luminous (i.e. SDSS z-band magnitudes of $18.74 \leqslant z_{A B} \leqslant 20.42$ ) objects from the SDSS main survey (e.g. Fan et al. 2006) and the objects from the SDSS southern deep imaging survey and the CFHQS (e.g. Jiang et al. 2009; Willott et al. 2007) that are one to two magnitudes fainter in the optical compared to the SDSS main survey. The MAMBO observations have reached a typical $1 \sigma$ sensitivity of $\sim 0.6 \mathrm{mJy}$ and 14 out of the 41 objects are detected at $\geqslant 3 \sigma$, yielding a detection rate of $34 \pm 9 \%$. This is consistent with the (sub)mm detection rates of optically selected quasars at redshifts 2 and 4 (Priddey et al. 2003; Omont et al. 2001, 2003; Carilli et al. 2001).

The FIR luminosities estimated with the $250 \mathrm{GHz}$ flux densities for the millimeterdetected $\mathrm{z} \sim 6$ quasars are a few $10^{12}$ to $10^{13} \mathrm{~L}_{\odot}$ (Wang et al. 2008, 2011). In the left panel of Figure 1, we compare the FIR-to-AGN bolometric luminosity relation of the $\mathrm{z} \sim 6$ quasars to the local optically selected PG quasars and a sample of IR luminous type I quasars hosted in ultra-luminous infrared galaxies (Hao et al. 2005). Most of the millimeter-detected $\mathrm{z} \sim 6$ quasars follow the shallower luminosity correlation trend defined by the IR quasars (Wang et al. 2008, 2011). This may suggest a starburst-dominant FIR emission in the millimeter bright quasars at $\mathrm{z} \sim 6$, similar to that found in the local IR quasars. We also calculate the average FIR emission by stacking the $250 \mathrm{GHz}$ measurements for the MAMBO non-detections in two quasar luminosity bins (i.e. optically bright, rest-frame $1450 \AA \mathrm{AB}$ magnitude $\mathrm{m}_{1450}<20.2$, and optically faint, $\mathrm{m}_{1450} \geqslant 20.2$, see Wang et al. 2011). The average FIR luminosity/upper limit for the non-detections is consistent with the trend defined by the local PG quasars.

Molecular CO (6-5) line emission has been detected in eleven of the millimeter-detected quasars at $\mathrm{z} \sim 6$ using the IRAM Plateau de Bure Interferometer (PdBI). The result indicates highly excited molecular gas on order of $10^{10} \mathrm{M}_{\odot}$ in the quasar host galaxies (Bertoldi et al. 2003b, Riechers et al. 2009; Carilli et al. 2007; Wang et al. 2010, 2011). The FIR and CO luminosities follow the relationship defined by actively star-forming galaxies at low and high redshifts (Riechers et al. 2006; Wang et al. 2010).

We are also searching for [C II] 158 micron fine structure line emission in our quasar sample. We have an on-going ALMA Cycle 0 program to look for this line in $\mathrm{z} \sim 6$ quasars with $250 \mathrm{GHz}$ continuum detections (Wang et al. 2012, in prep.). The data have been obtained for four of them in the extended configuration with typical resolution of $\sim 0.7^{\prime \prime}$ and all the four objects show clear detections. The [C II] luminosities are about 1 to $8 \times 10^{9} \mathrm{~L}_{\odot}$ and the [C II]-FIR luminosity ratios are of the order of $10^{-4}$, values that are comparable to those found for other high-z [C II]-detected quasars and about one order of magnitude lower than the typical value of star forming galaxies (e.g. Maiolino et al. 2009; Stacey et al. 2010; Wagg et al. 2012). The line velocity maps of three of them show indications of velocity gradients along the major axis direction. We plot the line intensity and velocity map for one of the detections, ULAS J131911.29+095051.4 at $\mathrm{z}=6.132$ (Mortlock et al. 2009), in the right panel of Figure 1. The line emission is 
marginally resolved by the $0.7^{\prime \prime} \times 0.5^{\prime \prime}$ beam, with a deconvolved size of $0.6^{\prime \prime} \times 0.3^{\prime \prime}$ (i.e. $3.5 \mathrm{kpc} \times 1.7 \mathrm{kpc}$, fitted to a two-dimensional Gaussian distribution).
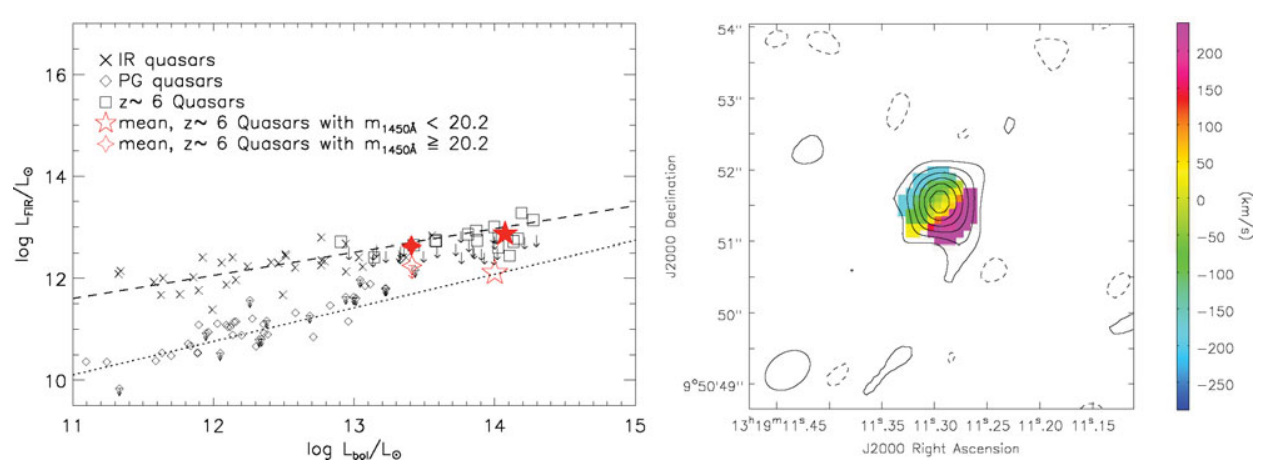

Figure 1. Left - The $\mathrm{L}_{\mathrm{FIR}}-\mathrm{L}_{\mathrm{bol}}$ correlation (Wang et al. 2008, 2011). The $250 \mathrm{GHz}$ detected $\mathrm{z} \sim 6$ quasars are plotted as black open squares and the arrows indicate $3 \sigma$ upper limits. The filled four-angle and five-angle stars are the average values for the optically faint $\left(\mathrm{m}_{1450} \geqslant 20.2\right)$ and bright $\left(\mathrm{m}_{1450}<20.2\right) 250 \mathrm{GHz}$ detections, respectively, and the open ones shows the stacking average of the non-detections. The local IR and PG quasars from Hao et al. (2005) are plotted as cross and diamonds with arrows denoting upper limits in $\mathrm{L}_{\mathrm{FIR}}$. The dashed line represent the linear regression of $\log \left(\mathrm{L}_{\mathrm{FIR}}\right)=0.45 \log \left(\mathrm{L}_{\mathrm{bol}}\right)+6.62$ derived with the local IR quasars and the (sub)mm detected quasars at high redshift (Wang et al. 2011), and the dotted line shows the relationship of $\log \left(\mathrm{L}_{\mathrm{FIR}}\right)=0.66 \log \left(\mathrm{L}_{\mathrm{bol}}\right)+2.8$ from the local PG quasars(Wang et al. 2008). Right - ALMA [C II] line velocity-integrated map (contours) and intensity-weighted velocity map (color) of the $\mathrm{z}=6.132$ quasar $\mathrm{J} 1319+0950$ (Wang et al. 2012, in prep.). The contours are $[-2,2,3,4,6,8,10,12] \times 0.18 \mathrm{Jy}_{\text {beam }}{ }^{-1} \mathrm{~km} \mathrm{~s}^{-1}$. The [C II] line emission is marginally resolved by the $0.7^{\prime \prime} \times 0.5^{\prime \prime}$ beam, with a source size of $(0.76 \pm 0.05)^{\prime \prime} \times(0.74 \pm 0.04)^{\prime \prime}$ (deconvolved size of $(0.6 \pm 0.1)^{\prime \prime} \times(0.3 \pm 0.2)^{\prime \prime}$, fitted using the IMFIT package in CASA $)$. The velocity map shows clear velocity gradient along the southwest-northeast direction.

\section{Discussion: star formation in the millimeter bright $\mathrm{z} \sim 6$ quasars}

The detections of strong dust continuum, molecular CO and [C II] fine structure line emission from the millimeter bright $\mathrm{z} \sim 6$ quasars strongly suggest active star formation in their host galaxies. In particular, the [C II] detections and the line velocity maps from our ALMA observation suggest a nuclear starburst disk over the central few kpc region. If we conservatively assume that $50 \%$ of the FIR emission is powered by host galaxy star formation, the estimated star formation rates (SFR) in the quasar host galaxies are about 600 to $2000 \mathrm{M}_{\odot} \mathrm{yr}^{-1}$ (adopting a standard Salpeter initial mass function, Kennicutt 1998). These together with the molecular gas masses measured from the CO line emission yield gas depletion time scales of $\tau_{\text {dep }}=\mathrm{M}_{\mathrm{gas}} / \mathrm{SFR} \sim 1-3 \times 10^{7} \mathrm{yr}$.

\section{Summary}

We summarize recent millimeter observations of the sample of quasars at $\mathrm{z} \sim 6$. About $30 \%$ of these objects have been detected in strong $250 \mathrm{GHz}$ dust continuum and molecular CO line emission, and our ongoing ALMA observations also detected bright [C II] fine structure line emission in the central few kpc region of the quasar host galaxies. The results suggest massive star formation in the quasar host galaxies, which is in good agreement with the picture of supermassive black hole-galaxy co-evolution at their earliest evolutionary epoch. Further high-resolution imaging of the dust, molecular, and atomic 
line emission in these objects (e.g. with Cycle 1 and the full configuration of ALMA) will fully probe the gas distribution, star formation rate surface density, star formation efficiency, and dynamical properties of the spheroidal quasar stellar bulges and address the SMBH-bulge relationships and quasar-galaxy evolution at the highest redshift.

This work is based on observations carried out with the Max Planck Millimeter Bolometer Array (MAMBO) on the IRAM 30m telescope, the Plateau de Bure Interferometer, and ALMA (NRAO). IRAM is supported by INSU/CNRS (France), MPG (Germany) and IGN (Spain). The National Radio Astronomy Observatory (NRAO) is a facility of the National Science Foundation operated under cooperative agreement by Associated Universities, Inc. This paper makes use of the following ALMA data: ADS/JAO.ALMA\# 2011.0.00206.S . ALMA is a partnership of ESO (representing its member states), NSF (USA) and NINS (Japan), together with NRC (Canada) and NSC and ASIAA (Taiwan), in cooperation with the Republic of Chile. The Joint ALMA Observatory is operated by ESO, AUI/NRAO and NAOJ.

\section{References}

Bertoldi, F., Carilli, C. L., Cox, P. et al. 2003a, A\&A, 406, L55

Bertoldi, F., Cox, P., Neri, R. et al. 2003b, A\&SA, 409, L47

Carilli, C. L., Bertoldi, F., Rupen, M. P. et al. 2001, ApJ, 555, 625

Carilli, C. L., Neri, R., Wang, R. et al. 2007, ApJ, 666, L9

Fan, X., Strauss, M. A., Richards, G. T., et al. 2006, AJ, 131, 1203

Hao, C. N., Xia, X. Y., Mao, S., Wu, H., \& Deng, Z. G. 2005, ApJ, 625, 78

Jiang, L., Fan, X., Bian, F. et al. 2009, AJ, 138, 305

Kennicutt, R. C. 1998, ARAESA, 36, 189

Kurk, J. D., Walter, F., Fan, X. et al. 2007, ApJ, 669, 32

Maiolino, R., Cox, P., Caselli, P. et al. 2005, A\&A, 440, L51

Maiolino, R., Caselli, P., Nagao, T., Walmsley, M., De Breuck, C., \& Meneghetti, M. 2009, A\&̊A, $500, \mathrm{~L} 1$

Maiolino, R., Gallerani, S., Neri, R. et al. 2012, MNRAS, 425, L66

Mortlock, D. J., Patel, M., Warren, S. J. et al. 2009, A\&SA, 505, 97

Omont, A., Cox, P., Bertoldi, F. et al. 2001, A $\mathscr{S} A, 374,371$

Omont, A., Beelen, A., Bertoldi, F., McMahon, R. G., Carilli, C. L., \& Isaak, K. G. 2003, A\&্A, 398,857

Petric, A. O., Carilli, C. L., Bertoldi, F. et al. 2003, AJ, 126, 15

Priddey, R. S., Isaak, K. G., McMahon, R. G., \& Omont, A. 2003, MNRAS, 339, 1183

Riechers, D. A., Walter, F., Carilli, C. L. et al. 2006, ApJ, 650, 604

Riechers, D. A., Walter, F., Bertoldi, F. et al. 2009, ApJ, 703, 1338

Stacey, G. J., Hailey-Dunsheath, S., Ferkinhoff, C. et al. 2010, ApJ, 724, 957

Venemans, B. P., McMahon, R. G., Walter, F. et al. 2012, ApJ, 751, L25

Wagg, J., Wiklind, T., Carilli, C. L. et al. 2012, ApJ, 752, L30

Walter, F., Bertoldi, F., Carilli, C. et al. 2003, Nature, 424, 406

Walter, F., Carilli, C. L., Bertoldi, F. et al. 2004, ApJ, 615, L17

Walter, F., Riechers, D., Cox, P. et al. 2009, Nature, 457, 699

Wang, R., Carilli, C. L., Wagg, J. et al. 2008, ApJ, 687, 848

Wang, R., Carilli, C. L., Neri, R. et al. 2010, ApJ, 714, 699

Wang, R., Wagg, J., Carilli, C. L. et al. 2011a, AJ, 142, 101

Willott, C. J., Delorme, P., Omont, A. et al. 2007, AJ, 134, 2435 